\title{
Non-adiabatic dynamics modeling framework for materials in extreme conditions
}

\author{
Hai Xiao $^{\mathrm{a}, 1}$, Andrés Jaramillo-Botero ${ }^{\mathrm{a}, 1, *}$, Patrick L. Theofanis ${ }^{\mathrm{b}}$, William A. \\ Goddard III ${ }^{\mathrm{a}, *}$ \\ ${ }^{a}$ Materials and Process Simulation Center, California Institute of Technology, Pasadena, \\ California 91125, USA \\ ${ }^{b}$ Intel Corporation, Santa Clara, California 95054, USA
}

\begin{abstract}
Modeling non-adiabatic phenomena and materials at extremes has been a long-standing challenge for computational chemistry and materials science, particularly for systems that undergo irreversible phase transformations due to significant electronic excitations. Ab initio and existing quantum mechanics approximations to the Schrödinger equation have been limited to groundstate descriptions or few excited electronic states, less than 100 atoms, and sub-picosecond timescales of dynamics evolution. Recently, the electron force field (eFF) introduced by ? presented a cost-efficient alternative to describe the dynamics of electronic and nuclear degrees of freedom. eFF describes an $\mathrm{N}$-electron wave function as a Hartree product of one-electron floating spherical Gaussian wave packets propagating via the time-dependent Schrödinger equation under a mixed quantum-classical Hamiltonian evaluated as sum of self- and pairwise potential interactions. Local Pauli potential corrections replace the need for explicit anti-symmetrization of total electronic wavefunction, a wavefunction kinetic energy term accounts for Heisenberg's uncertainty, and classical electrostatics complete the total eFF energy expression. However, due to the spherical symmetry of the underlying Gaussian basis functions, the original eFF formulation is limited to low-Z numbers with electrons of predominant $s$-character. To overcome this, we introduce here
\end{abstract}

\footnotetext{
*Corresponding authors.

Email addresses: ajaramil@caltech.edu (Andrés Jaramillo-Botero), wag@wag. caltech.edu (William A. Goddard III)

${ }^{1}$ These authors contributed equally to this work.
} 
a formal set of potential form extensions that enable accurate description of $p$-block elements in the periodic table. The extensions consist of a model representing the core electrons of an atom together with the nucleus as a single pseudo particle with wave-like behavior, interacting with valence electrons, nuclei, and other cores through effective core pseudopotentials (ECPs). We demonstrate and validate the ECP extensions for complex bonding structures, geometries and energetics of systems with $p$-block character (containing silicon, oxygen, carbon, or aluminum atoms and combination thereof) and apply them to study materials under extreme mechanical loading conditions.

Keywords: Materials in extreme conditions, Large scale non-adiabatic dynamics, wave-packet dynamics, electron force field (eFF), effective core pseudopotential (ECP), high-Z elements

PACS: 31.70.Hq, 32.80.Rm, 34.50.Gb

\section{Introduction}

The Born-Oppenheimer (BO) approximation, which decouples the nuclear and electronic motions, constitutes one of the fundamental assumptions for most of atomistic modeling techniques, ranging from first principle electronic structure methods, such as Hartree-Fock (HF) and density functional theory (DFT) for accurate description of potential energy surfaces (PES), to force field methods that enable molecular dynamics (MD) simulations of large scale systems through classical approximations of PES. However, the $\mathrm{BO}$ approximation breaks down for systems in extreme conditions where the electronic portion of the wavefunction contains contributions from many stationary states (?), such as those found at extremes of temperature, shock, radiation, etc. which cause irreversible material transformations, fatigue, embrittlement and ultimately failure.

Several methods have been developed to describe the coupling of nuclear and electronic motions, including surface hopping schemes (?) which rely on PES generated by high level ab initio methods, Ehrenfest dynamics with time-dependent HF/DFT engines (??), and fermionic dynamics (??) approaches. All these techniques are computationally expensive and impractical for performing long time scale dynamics of large scale non-adiabatic materials phenomena.

The electron force field (eFF) (?) was developed to overcome this limitation (see Fig. 1) and recent improvements to it (?) confirm its scalability and 
applicability to challenging problems including, but not limited to: explaining electronic phenomena during brittle fracture of silicon (?), understanding the mechanisms of Auger induced chemical decomposition (?), characterizing hydrostatic and dynamic shock Hugoniots for different materials (????), and tracking the dynamics of Coulomb explosion in silicon and diamond nanoparticles (?), among others (?).

Here, we present a formal extension to support higher $\mathrm{Z}$ elements in eFF using effective core pseudopotentials, and their validation on $\mathrm{C}, \mathrm{O}, \mathrm{Si}$, and $\mathrm{Al}$ based systems (energetics and geometries) using quantum mechanics.



Figure 1: eFF aims at long term and large scale non-adiabatic MD simulations in which the electronic wave function positions and sizes vary dynamically.

In the framework of eFF, nuclei are classical point charges and the total electronic wavefunction is represented by a Hartree product of one-electron floating spherical Gaussian (FSG) wave packets, Eq.(1), whose positions, $\vec{x}_{i}$, and sizes, $s_{i}$, are both dynamic variables.

$$
\Psi\left(\vec{r}_{i}\right) \propto \prod_{i} \exp \left[-\left(\frac{1}{s_{i}^{2}}-\frac{2 p_{s_{i}}}{s_{i}} \frac{i}{\hbar}\right)\left(\vec{r}_{i}-\vec{x}_{i}\right)^{2}\right] \exp \left[\frac{i}{\hbar} \vec{p}_{\vec{x}_{i}} \cdot \vec{r}_{i}\right]
$$

This representation leads to a rather simple electronic energy expression, $\langle\Psi|\hat{H}| \Psi\rangle$, consisting of the sum of single-particle kinetic energy and pairwise Coulomb energies. Additionally, pairwise spin-dependent Pauli corrections 
are introduced to locally compensate for the lack of explicit wavefunction anti-symmetrization. As a result, the electronic contribution to the total energy is evaluated as in classical force field methods. Furthermore, semiclassical equations of motion (EOM) for propagating the electronic wavefunction, as shown in Fig. 1, are derived from the time dependent Schrödinger equation with a local harmonic potential approximation. The combination of force field like energy evaluation and semi-classical EOM in eFF enables long term and large scale non-adiabatic MD simulations of low-Z systems, as demonstrated in previous work (?).

However, an intrinsic limitation of the all-electron FSG-based eFF described above emanates from the spherical symmetry of the underlying basis functions. For atoms with valence electrons of higher angular momenta, such as p-block elements, the FSG representation misses part of the interaction between the core and valence electrons, due to the absence of nodal structures.

Here, we present in Sec. 2 one approach to mitigate this problem, in the form of effective core pseudopotentials (ECP). This model form of ECP replaces the interaction between the core and the valence electrons with a potential energy given by their overlap. The corresponding parameters that define the ECP are obtained from first-principles quantum mechanics. In Sec. 3, we show that the resulting ECP formulation appropriately captures part of the missing $p$-character of FSG valence electrons, which leads to a correct description of complex bonding structures (e.g. multiple bonds and lone pairs) for systems containing $p$-block elements of the second and third row of the periodic table. In particular, we demonstrate parameters for silicon, aluminum, carbon, oxygen and binary combination $\mathrm{SiC}$, and example applications using the open source implementation (?) available in the parallel molecular dynamics simulator LAMMPS (?).

\section{Effective core pseudopotential (ECP) models in eFF}

The full eFF Hamiltonian, shown in Eq.(2), has a standard description for electrostatic interactions between a set of zero-dimensional points and Gaussian charges which include, nucleus-nucleus $\left(E_{N N}\right)$, electron-electron $\left(E_{e e}\right)$, and nucleus-electron $\left(E_{N e}\right)$. In addition to the electrostatics, eFF introduces quantum effects through an electron kinetic energy from the Gaussian $\left(E_{K E}\right)$ and a spin-dependent Pauli repulsion potential term $\left(E_{P R}\right)$ between Gaussians (further details can be found in previous work (??)). 
$U(R, r, s, \sigma)=E_{N N}\left(R_{N N}\right)+E_{N e}\left(R_{N e}, s\right)+E_{e e}\left(r_{e e}, s\right)+E_{K E}(s)+E_{P R}(\sigma, s)$

where $R_{N N}, R_{N e}$, and $r_{e e}$ correspond to the inter-nucleus, nucleus-electron and inter-electron distances, respectively, $s$ to the electron radius, and $\sigma$ to the electron spin.

The eFF-ECP scheme presented here requires reformulating and parameterizing the Pauli energy term, $E_{P R}$, for pseudo particles with Gaussian charge replacing the core electrons and the nucleus, and adjusting the classical electrostatic energies between the pseudo-core and valence electrons (core-elec), nuclei (core-nuc), and other pseudo-core (core-core) particles as,

$$
\begin{array}{r}
E_{\text {core-elec }}=\sum_{i, j} \frac{Z_{i} Z_{j}}{R_{i j}} \operatorname{Erf}\left(\frac{\sqrt{2} R_{i j}}{\sqrt{s_{\text {core }, i}^{2}+s_{\text {elec }, j}^{2}}}\right), \\
E_{\text {core-nuc }}=\sum_{i, j} \frac{Z_{i} Z_{j}}{R_{i j}} \operatorname{Erf}\left(\frac{\sqrt{2} R_{i j}}{\sqrt{s_{\text {core }, j}^{2}}}\right), \\
E_{\text {core-core }}=\sum_{i<j} \frac{Z_{i} Z_{j}}{R_{i j}} \operatorname{Erf}\left(\frac{\sqrt{2} R_{i j}}{\sqrt{s_{\text {core }, i}^{2}+s_{\text {core }, j}^{2}}}\right)
\end{array}
$$

where $Z$ is the particle charge.

The new Pauli potentials in this model ECP representation are designed, according to the relation $E_{P R} \propto S^{2}$, where $S$ is the overlap between two Gaussians: one representing the core and the other an interacting valence electron. By choice, two different types of overlaps are defined in this ECP representation as:

1. an $s$-s overlap, for an $s$-type valence electron, and

2. an $s-p$ overlap, for an $p$-type valence electron.

The corresponding functional forms are derived as,

$$
E_{P R_{s-s}}=\mathbf{a} \exp \left(-\frac{\mathbf{b} r^{2}}{\mathbf{c}+s^{2}}\right)
$$




$$
E_{P R_{s-p}}=\mathbf{a}\left(\frac{2}{\mathbf{b} / s+s / \mathbf{b}}\right)^{5}(r-\mathbf{c} s)^{2} \exp \left(-\frac{\mathbf{d}(r-\mathbf{c} s)^{2}}{\mathbf{e}+s^{2}}\right)
$$

where $r$ in Eq.(4) is the distance between the $s$-type pseudo-core and an interacting $s$-type valence electron, and in Eq.(5) it corresponds to the distance between the $s$-type pseudo-core and the $s$-type Gaussian representing one of the lobes of a $p$-type valence electron (see Fig. 2). $s$ is the size of the corresponding valence electron, a corresponds to the pseudo-core wave function amplitude, $\mathbf{b}$ in Eq.(4) and $\mathbf{d}$ in Eq.(5) to the pseudo-core wavefunction decay factor, c in Eq.(4) and e in Eq.(5) to the square of effective pseudo-core particle size (not to be confused with the core size used in Coulomb energies, in Eq.(3), which is also a parameter). For the $s$ - $p$ case c corresponds to an off-center measure and $\mathbf{b}$ to a second effective size that adjusts the overlap amplitude.
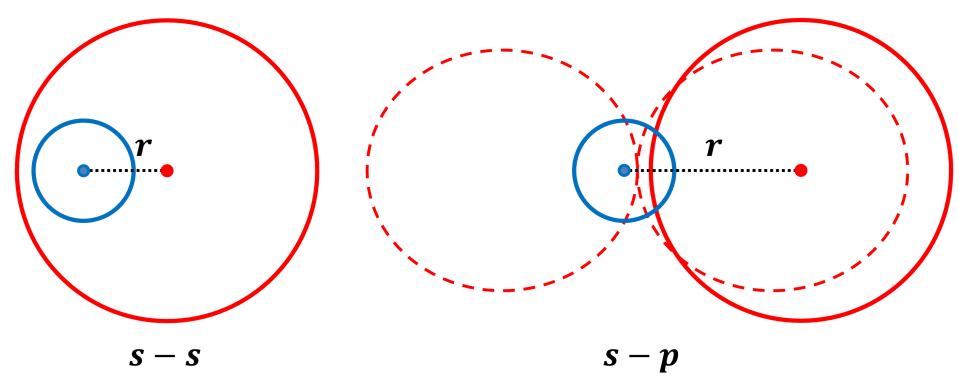

Figure 2: Illustration of distances used in the two functional types of ECP, where blue circles represent core electrons and red valence electrons. Note that the center of real $p$-type Gaussian is the nodal point.

Eq.(4) and Eq.(5) are derived from the square of the overlap between spherical Gaussians used in eFF,

$$
\phi_{i}(\vec{r})=\left(\frac{\sqrt{2}}{\sqrt{\pi} s_{i}}\right)^{3 / 2} \exp \left[-\frac{\left(\vec{r}-\vec{r}_{i}\right)^{2}}{s_{i}^{2}}\right]
$$

neglecting phase factors associated with dynamics (one Gaussian representing a valence electron and the other an atom's core set of electrons), with index $i=1,2$ for core and valence electrons, respectively. 
The square of overlap between $s$-type core and $s$-type valence is given by

$$
S^{2}=\left(\frac{2}{s_{1} / s_{2}+s_{2} / s_{1}}\right)^{3} \exp \left[-\frac{2 r_{12}^{2}}{s_{1}^{2}+s_{2}^{2}}\right]
$$

For a $p$-type Gaussian representing the valence electron,

$$
\phi_{2}(\vec{r})=\left(\frac{\sqrt{2}}{\sqrt{\pi} s_{i}}\right)^{3 / 2} \frac{2}{s_{2}}\left(\vec{r}-\vec{r}_{2}\right) \exp \left[-\frac{\left(\vec{r}-\vec{r}_{2}\right)^{2}}{s_{2}^{2}}\right]
$$

the square of overlap between $s$-type core and $p$-type valence is given by

$$
S^{2}=\left(\frac{2}{s_{1} / s_{2}+s_{2} / s_{1}}\right)^{5} \frac{r_{12}^{2}}{s_{1}^{2}} \exp \left[-\frac{2 r_{12}^{2}}{s_{1}^{2}+s_{2}^{2}}\right]
$$

In order to preserve the simple form of the semi-classical EOM in eFF, a spherical Gaussian is used to represent one of the two lobes of the $p$-type valence electron. Therefore, the center of an $s$-type valence electron is that of the spherical Gaussian, while that of a $p$-type valence electron, which is the nodal point, and its offset is determined by $s_{2} / \sqrt{2}$, with the reasonable assumption that the center of spherical Gaussian is at exactly the cusp of the lobe. So, Eq.(9) is recast into

$$
S^{2}=\left(\frac{2}{s_{1} / s_{2}+s_{2} / s_{1}}\right)^{5} \frac{\left(r_{12}-s_{2} / \sqrt{2}\right)^{2}}{s_{1}^{2}} \exp \left[-\frac{2\left(r_{12}-s_{2} / \sqrt{2}\right)^{2}}{s_{1}^{2}+s_{2}^{2}}\right]
$$

Following the design of $E_{P R} \propto S^{2}$, two types of ECP formulation can be constructed,

$$
\begin{gathered}
E_{P R_{s-s}}=a\left(\frac{2}{s_{1} / s_{2}+s_{2} / s_{1}}\right)^{3} \exp \left[-\frac{2 r_{12}^{2}}{s_{1}^{2}+s_{2}^{2}}\right] \\
E_{P R_{s-p}}=a\left(\frac{2}{s_{1} / s_{2}+s_{2} / s_{1}}\right)^{5} \frac{\left(r_{12}-s_{2} / \sqrt{2}\right)^{2}}{s_{1}^{2}} \exp \left[-\frac{2\left(r_{12}-s_{2} / \sqrt{2}\right)^{2}}{s_{1}^{2}+s_{2}^{2}}\right]
\end{gathered}
$$

Equations (4) and (5) are directly obtained from Eq.(11) and Eq.(12), respectively, by defining and replacing with the corresponding parameter variables. 
In section 3 we present optimized eFF-ECP parameters for aluminum $(s-$ $s)$, silicon $(s-s)$, carbon $(s-p)$ and oxygen $(s-p)$ and validate them on example applications. All parameters were optimized against diverse training sets of geometries and energetics obtained from quantum mechanical (QM) calculations of small representative molecules. A two-step parameter optimization scheme was used, consisting of: 1) a genetic algorithm based search to determine the most probable global basin, and 2) a local conjugate gradient based optimization within the local search space of the GA-determined basin in order to remove any randomness in the solution. The GA was configured with tournament selection and uniform mutation over the predefined range for each parameter and evaluation was done via a fitness function of geometric and energetic RMSDs (root mean square deviations) from the QM reference set. Further details on the parameter optimization framework can be found in (?).

\section{Results and Discussions}

Table 1 summarizes the eFF-ECP parameters for Aluminum (Al), Silicon $(\mathrm{Si})$, Carbon $(\mathrm{C})$, and Oxygen $(\mathrm{O})$. $\mathrm{Al}$ and $\mathrm{Si}$ can be accurately described using the $s$ - $s$ ECP form in Eq. (4), i.e. $\mathrm{E}_{P R_{s-s}}$, while $\mathrm{C}$ and $\mathrm{O}$ require the higher-order $s$ - $p$ form in Eq. (5), i.e. $\mathrm{E}_{P R_{s-p}}$, due to their more complex and dominant $p$-type interactions, including multiple bonds and lone pairs.

The core radii were initially estimated by calculating the sizes of corresponding all-electron ions (i.e., $\mathrm{Al}^{3+}, \mathrm{Si}^{4+}, \mathrm{C}^{4+}$ and $\mathrm{O}^{6+}$ ) with the all-electron $\mathrm{eFF}$, and then optimized to fit the correct electrostatics in the ECP description. In total, 4 parameters were optimized for the corresponding $s$ - $s$ ECP cases, and 6 for the corresponding $s-p$ cases.

Table 1: eFF-ECP parameters determined for different $2^{\text {nd }}$ and $3^{\text {rd }}$ row $p$-block elements.

\begin{tabular}{lccccccc}
\hline & Element & $\mathbf{a}$ & $\mathbf{b}$ & $\mathbf{c}$ & $\mathbf{d}$ & $\mathbf{e}$ & $s_{\text {core }}$ (bohr) \\
\hline$s-s$ (Eq. (4)) & & & & & & & \\
& $\mathrm{Al}$ & 0.486000 & 1.049000 & 0.207000 & & & 1.660000 \\
& $\mathrm{Si}$ & 0.320852 & 2.283269 & 0.814857 & & & 1.691398 \\
\hline$s-p$ (Eq. (5)) & & & & & & & \\
& $\mathrm{C}$ & 22.721015 & 0.728733 & 1.103199 & 17.695345 & 6.693621 & 0.621427 \\
& $\mathrm{O}$ & 25.080199 & 0.331574 & 1.276183 & 12.910142 & 3.189333 & 0.167813 \\
\hline
\end{tabular}


Hybrid (binary, tertiary, etc.) systems may be modeled using the parameters above, nonetheless, increased accuracy may be achieved by re-optimizing these parameters with QM cases that include all expected interactions. This will be exemplified here for silicon carbide.

\subsection{Aluminum and Silicon with s-s ECP}

The effective core in aluminum is defined as a pseudo particle with wavelike properties, consisting of a nucleus and ten fixed electrons corresponding to the $1 s^{2} 2 s^{2} 2 p^{6}$ configuration. This effective core plus three explicit valence electrons $\left(3 s^{2} 3 p^{1}\right)$ complete the 4-particle eFF-ECP model representation of aluminum.

ECP parameters for aluminum were determined from ground state $f c c$ bulk aluminum, lattice parameters and bulk modulus experimental values $\left(a_{e x p}=4.05 \AA\right.$ and $\left.\mathrm{B}_{\exp }=76 \mathrm{GPa}\right)$, and finite aluminum hydride molecules, including those compared in Table 2. The resulting eFF-ECP lattice parameters and bulk modulues are $a_{e F F-E C P}=4.05 \AA$ and $\mathrm{B}_{e F F-E C P}=108 \mathrm{GPa}$, in contrast to the all-electron eFF values of $a_{e F F}=4.23 \AA$ and $\mathrm{B}_{e F F}=44$ GPa.


Figure 3: $\mathrm{Al}_{2} \mathrm{H}_{6}$ and $\mathrm{AlH}_{3}$ molecular geometries using eFF-ECP.

Aluminum clusters constitute an interesting system to understand different phenomena at the nanoscale, such as the effect of higher hydrogen contents. Consequently, we calculated emergent properties for $A_{n} H_{3 n}$ aluminum hydrige clusters of $n=1 . .5$, including the bond energies as a function of cluster size as shown in Fig. 4. Angles in degrees, distances in pico-meters, and energies in $\mathrm{Kcal} / \mathrm{mol}$.

The original all-electron eFF does not capture aluminum hydride bond energies for clusters with more than one aluminum atom (i.e. $n=1$ ), while eFF-ECP accurately tracks DFT with LCAO+B3LYP (Linear Combination 
Table 2: Comparison of eFF-ECP, all electron eFF and DFT calculations on aluminum hydrides (see Figure 3).

\begin{tabular}{lcccccc}
\hline \multirow{2}{*}{ Feature } & \multicolumn{3}{c}{$\mathrm{Al}_{2} \mathrm{H}_{6}$} & \multicolumn{3}{c}{$\mathrm{AlH}_{3}$} \\
& $\mathrm{~B} 3 \mathrm{LYP} / \mathrm{MO} 6$ & $\mathrm{eFF-ECP}$ & $\mathrm{eFF}$ & $\mathrm{B} 3 \mathrm{LYP} / \mathrm{MO} 6$ & $\mathrm{eFF}-\mathrm{ECP}$ & $\mathrm{eFF}$ \\
\hline $\mathrm{d}_{A l-A l}$ & $258.3 / 261.382$ & 267.142 & 220.0 & & & \\
$\mathrm{~d}_{A l-H}$ & $157.7 / 157.3$ & 148.241 & 159.7 & $158.4 / 158.1$ & 151.932 & 160.4 \\
$\mathrm{~d}_{A l-H_{\text {bridge }}}$ & $174.5,170.9 / 174.4,174.2$ & 178.306 & 161.6 & & & \\
$\theta_{A l-A l-H_{\text {bridge }}}$ & $42.105,41.051 / 41.386,41.441$ & 41.486 & 47.111 & & 120.0 & 120.0 \\
$\theta_{H-A l-H}$ & $127.493 / 128.168$ & 128.647 & 116.71 & 120.0 & & \\
$\theta_{H-A l-H_{\text {bridge }}}$ & $109.3 / 109.042$ & 108.94 & 110.858 & & & \\
$\mathrm{E}_{A l_{2} H_{6} \rightarrow 2 \text { Al H }_{3}}$ & $32.74698 / 34.72389$ & 33.3571 & 133.8163 & & & \\
\hline
\end{tabular}

of Atomic Orbitals method and B3LYP hybrid functional) up to $n=5$ in cyclic and linear polymeric structures (see 2). What's interesting, is that eFF-ECP accurately reproduces the planar structures up to $n=5$ (because the $3 s$ atomic state behave like a core level due to the large energy separation between the $3 s$ and the $3 p$ states). eFF-ECP confirms the origin of increased stability of these cyclic and linear polymeric molecules comes from the stronger $\mathrm{Al}-\mathrm{H}$ bonds, versus the $\mathrm{Al}-\mathrm{Al}$ bonds in the size range $(n=1 . .5)$ as reported by ?. Furthermore, we believe it can capture the changes in bonding nature, from $p$ - to $s p$ - hybridization as the clusters grow in three dimensions and towards a lower total energy.

The effective core in silicon has the same composition as the pseudo-core particle for aluminum, yet with four explicit valence electrons corresponding to the $3 s^{2} 3 p^{2}$ orbitals complete its 5 -particle eFF-ECP model representation. The ECP parameters for silicon were determined from first principle calculations on small silicon hydride motifs, including: $\mathrm{SiH}_{3}-\mathrm{H}$ and $\mathrm{H}_{3} \mathrm{Si}-$ $\mathrm{SiH}_{3}$ energies, Si-H and Si-Si bond lengths, bulk lattice constant, and bond dissociation energies for several silicon and silicon hydride compounds (as described in (?)).

The silicon eFF-ECP parameters were validated on emergent material properties, such as ionization potential, $\mathrm{Si}_{2} \mathrm{H}_{6}$ rotational barriers, bulk modulus, and yield strength and used to describe the non-adiabatic quantum dynamics during brittle fracture in silicon crystal in previous work (?). The silicon eFF-ECP simulations reproduce the correct response of the crack tip velocity to the threshold critical energy release rate, a feat that is in- 




Figure 4: Comparison of cluster bond energies for different $\mathrm{Al}_{n} \mathrm{H}_{3} n$ clusters. QM data from $(?)$ 




Figure 5: Crack tip velocity versus reduced load for 111 fracture from eFF-ECP, compared to experimental, ReaxFF+Tersoff simulations, and DCET and EDIP data - normalized by the Griffith critical load value. The grey line is a visual guide. From (?).

accessible to quantum mechanics methods or conventional force-field-based molecular dynamics (see Fig. 5), and describe the crack induced voltages, current bursts, and charge carrier production observed experimentally during fracture. This led to an explanation of how strain-induced surface rearrangements and local heating cause ionization of electrons at the fracture surfaces.

Our simulations reveal both local field-induced ionization and thermal ionization as a direct result of fracture, which leads to crack induced voltages across the fractured surfaces. We find that electron ionization is precipitated by the passing of the crack front (Fig. 6a). Ionized electrons are excited by $5 \mathrm{eV}$, making them sufficiently energetic to escape the Si-surface barrier (Fig. 6b). The initial excitation promotes the electrons to unbound states, but they subsequently relax to $4.1 \mathrm{eV}$ above the ground state, well into the $\mathrm{Si}$ conduction band. An increase in potential energy causes ionization, primarily via heterolytic bond cleavage across the crack. In rare instances a heterolytic cleavage creates an anion on one crack face and a cation on the other crack face. As dangling bonds form $2 \times 1$ surface dimers, the excess electron causes Pauli exclusion clashes with adjacent surface pairs (Fig. 6c) and the ionized electron's radius decreases to reduce it's overlap with nearby 
same-spin electrons (Fig. 6d). The spin clashing forces the electron further from the surface and the electron delocalizes (Fig. 6e). Ultimately it relaxes and settles into the conduction band. $80 \pm 10 \%$ of ionized electrons are ionized because of local field effects.



(a)



(d)


(b)



(e)



(c)



Figure 6: (a) High strain rate cracking of Si leads to (b) surface electron ionization, (c) spin exchange, (d) increased electronic wavefunction kinetic energy for neighboring same-spin electrons, (e) and spin-clashing of surface electrons during $2 \times 1$ surface dimer reconstruction, which leads to the electron emissions observed experimentally.

\subsection{Carbon with s-p ECP}

The all-electron eFF formulation performs well in describing saturated hydrocarbons (?), yet it is inaccurate for unsaturated systems and fails at describing complex bonding structures, including multiple bonds and lone 
pairs, among others. Here we demonstrate the use of the $\mathrm{E}_{P R_{s-p}}$ eFF-ECP (Eq. 5) to overcome these issues, for the most part caused by increasingly non-spherical character in electrons.

In preparing the carbon eFF-ECP parameters, we set out to: retain the all-electron eFF performance for saturated hydrocarbons, improve the bonding energy of $\mathrm{C}-\mathrm{C}$, and enable complex bonding descriptions. To this end, the prepared ECP training set includes geometries for: $\mathrm{CH}_{4}, \mathrm{C}_{2} \mathrm{H}_{6}, \mathrm{CH}_{2}\left(\mathrm{CH}_{3}\right)_{2}$, $\mathrm{CH}\left(\mathrm{CH}_{3}\right)_{3}, \mathrm{C}_{2} \mathrm{H}_{4}, \mathrm{C}_{2} \mathrm{H}_{2}$ and $\mathrm{C}\left(\mathrm{CH}_{3}\right)_{4}$; and the bonding energy of $\mathrm{C}-\mathrm{C}$ in $\mathrm{C}_{2} \mathrm{H}_{6}$. Table 3 includes the comparison of optimized results from eFF-ECP with the all-electron eFF representation and experiments.

In general, eFF-ECP improves the dependence of $\mathrm{C}-\mathrm{C}$ and $\mathrm{C}-\mathrm{H}$ bond lengths on the size of saturated hydrocarbons. The all-electron eFF predicts a large increase in both bond lengths as the hydrocarbon size increases, while eFF-ECP captures the correct trend. The $\sim 10 \%$ reduction in the $\mathrm{C}-\mathrm{C}$ bond length for bulk diamond is one example that confirms this (i.e. it is not part of the training set). On the other hand, the overestimation of $\mathrm{C}-\mathrm{C}$ bonding energy in ethane is corrected, from $163.5 \mathrm{kcal} / \mathrm{mol}$ in the all-electron eFF to an experimentally accurate value of $89.7 \mathrm{kcal} / \mathrm{mol}$ in eFF-ECP.

Table 3: Comparison of eFF-ECP and eFF on geometries of molecular and bulk systems.

\begin{tabular}{cccccccccc}
\hline \multirow{2}{*}{ Species } & \multicolumn{3}{c}{$d_{C C}(\mathrm{pm})$} & \multicolumn{3}{c}{$d_{C H}(\mathrm{pm})$} & \multicolumn{3}{c}{$\Theta$ (degree) } \\
& exact & eFF-ECP & eFF & exact & eFF-ECP & eFF & exact & eFF-ECP & eFF \\
\hline $\mathrm{CH}_{4}$ & & & & 109.4 & 105.6 & 114.3 & 109.5 & 109.5 & 109.5 \\
$\mathrm{C}_{2} \mathrm{H}_{6}$ & 153.6 & 152.0 & 150.1 & 109.1 & 106.5 & 117.3 & 110.9 & 111.3 & 110.8 \\
$\mathrm{CH}_{2}\left(\mathrm{CH}_{3}\right)_{2}$ & 152.6 & 152.6 & 151.3 & 109.6 & 107.6 & 122.9 & 109.5 & 110.6 & 107.9 \\
$\mathrm{CH}\left(\mathrm{CH}_{3}\right)_{3}$ & 152.5 & 153.0 & 152.9 & 110.8 & 108.7 & 142.4 & 109.4 & 109.9 & 101.8 \\
$\mathrm{C}\left(\mathrm{CH}_{3}\right)_{4}$ & 153.4 & 153.2 & 157.3 & 111.4 & 106.7 & 117.8 & & & \\
$\mathrm{H}_{2} \mathrm{C}=\mathrm{CH}_{2}$ & 133.9 & 137.7 & 151.7 & 108.6 & 97.2 & 108.9 & & & \\
$\mathrm{HC} \equiv \mathrm{CH}$ & 120.3 & 115.4 & 138.3 & 106.3 & 91.8 & 105.2 & & & \\
Diamond & 154.5 & 155.1 & 168.1 & & & & & & \\
\hline
\end{tabular}

eFF-ECP now describes both single and multiple hydrocarbon bonds in a consistent manner, i.e. the bond length decreases as the bond order increases. eFF-ECP also improves the stability of multiple bonds. For example, the hydrogenation energy of a double bond in ethene (not in the training set) is predicted to be exactly the same as the experimental value of $38.2 \mathrm{kcal} / \mathrm{mol}$, while the all-electron eFF value results in $141.6 \mathrm{kcal} / \mathrm{mol}$. Furthermore, 
rather than the non-planar diradical conformation given by all-electron eFF, eFF-ECP predicts the correct planar conformation for benzene molecule, although with alternating single and double bonds, which is inevitable from the localized nature of eFF framework.

Fig. 7 shows a few molecules with conjugate double bonds, all of which are described with correct conformation by eFF-ECP. A more challenging system is the graphite bulk structure, which involves capturing the correct London dispersion energies. eFF-ECP predicts surprisingly good lattice parameters, with $a=2.550 \AA$ and $c=6.943 \AA$ (vs. the experimental value of $a=2.461 \AA$ and $c=6.708 \AA$ ). An interesting side effect is that the subtle London dispersion energies that stabilize graphite layers along the $c$ axis is seemingly well captured with the eFF-ECP framework, which is not expected by design, while conventional DFT fails to capture this (without explicit dispersion corrections), as shown in Fig. 8. This is likely due to multipole interactions, arising from the fact that the electrons, having different sizes for different spins (like in the benzene molecule), are arranged in an alternating way on both sides of each layer and facing sides of two neighboring layers.

To test the non-adiabatic dynamics modeling capabilities of the C-ECP, we applied this force field to study and understand the effect of induced energy excitations on the valence electrons of silicon-hydrogen bonds of a passivation layer in a diamond film, in the presence of an atomic hydrogen gas. The idea being that surface selective chemistry may be achieved via excitation energy transfer from external sources, for example, an electron beam from an electron stimulated desorption (ESD) apparatus, onto resonant modes on the material surface; in the case reported here, the valence electrons that participate in the Si-H surface bonds. A $2 \times 1$ dimerized and $\mathrm{H}$-capped diamond surface model slab was prepared and equilibrated to a temperature of $373 \mathrm{~K}$, and subsequently combined with an atomic $\mathrm{H}$ gas equilibrated to $15,000 \mathrm{~K}$ in a single non-equilibrium NVE ensemble run (constant number of particles, volume and number of particles). Two separate systems were run concurrently, one in which the surface electrons in the $\mathrm{C}-\mathrm{H}$ bonds were manually excited by a step-function of $5 \mathrm{eV}$ at $t=0$ (i.e. to achieve the manual excitation, the electron radii in the $\mathrm{Si}-\mathrm{H}$ bonds of the passivation layer were reduced from 1.65 to 1.43 Bohr, which is equivalent to an energy input slightly below diamond's bandgap energy) and one in which no manual excitation was introduced on the surface bonds. For the system that was manually excited, etching events were observed within 80 femtoseconds, while the un-excited systems ran for more than 1 picosecond without any hydrogen 




(a) Benzene (planar)



(c) Naphthalene (planar)



(b) Cyclooctatetraene (nonplanar)



(d) Pyrene (planar)

Figure 7: Gallery of molecules with conjugate double bonds. eFF-ECP is able to predict correct conformations for all of them. The red/blue colors stand for electronic spin up or down, respectively.

etching events. The enhanced hydrogen abstraction mechanism involves a surface passivating hydrogen being knocked out by the excitation energy transfer to the associated $\mathrm{C}-\mathrm{H}$ surface bond electrons from a radical hydrogen atom of the plasma phase. This is followed by a fast recombination of the loose surface hydrogen and the radical hydrogen from the plasma phase into an ejected $\mathrm{H}_{2}$ molecule (shown graphically in Fig. 9.)

\subsection{Oxygen with s-p ECP}

One of the prominent features characterizing the chemistry of oxygen is the presence of lone pairs on it while bonding. These have a predominant $p$-character, and all-electron eFF fails in describing such systems, qualitatively and quantitatively. For example, the all-electron eFF predicts the water molecule to be linear, instead of bent. With the $s-p$ ECP type, eFFECP is shown to handle lone pairs smoothly. Interestingly, the lone pair is represented by an open shell like configuration, i.e., the size of electron with 



(b)

Figure 8: (a) Comparison of various methods, including quantum Monte Carlo (QMC) (?) and dispersion-corrected PBE-lg (?), on the binding curve along the dispersion $c$ axis of graphite. (b) Illustration of graphite described with eFF-ECP.

one spin being larger than that of electron with the other spin, as show in Fig. 10, illustrated with water molecule.

The training set for $\mathrm{O}$ ECP includes $\mathrm{Li}_{2} \mathrm{O}, \mathrm{Si}_{2} \mathrm{H}_{6} \mathrm{O}, \mathrm{Si}_{3} \mathrm{H}_{8} \mathrm{O}_{2}, \mathrm{Si}_{3} \mathrm{H}_{6} \mathrm{O}_{2}$, $\mathrm{SiH}_{3} \mathrm{OH}$ and $\mathrm{H}_{2} \mathrm{O}$, covering a variety of bonding characters, reflected by the various oxygen-centered angles (see Table 4). This is because, in addition to describing lone pairs, we wanted eFF-ECP to describe the subtle coupling between interacting lone pairs and bonding character. We validate that when oxygen atoms forms purely ionic bonds, such as in $\mathrm{Li}_{2} \mathrm{O}$, the electrostatic repulsion between two cations dominates, leading to a linear molecular conformation; while as the degree of covalent bonding increases, the repulsion 


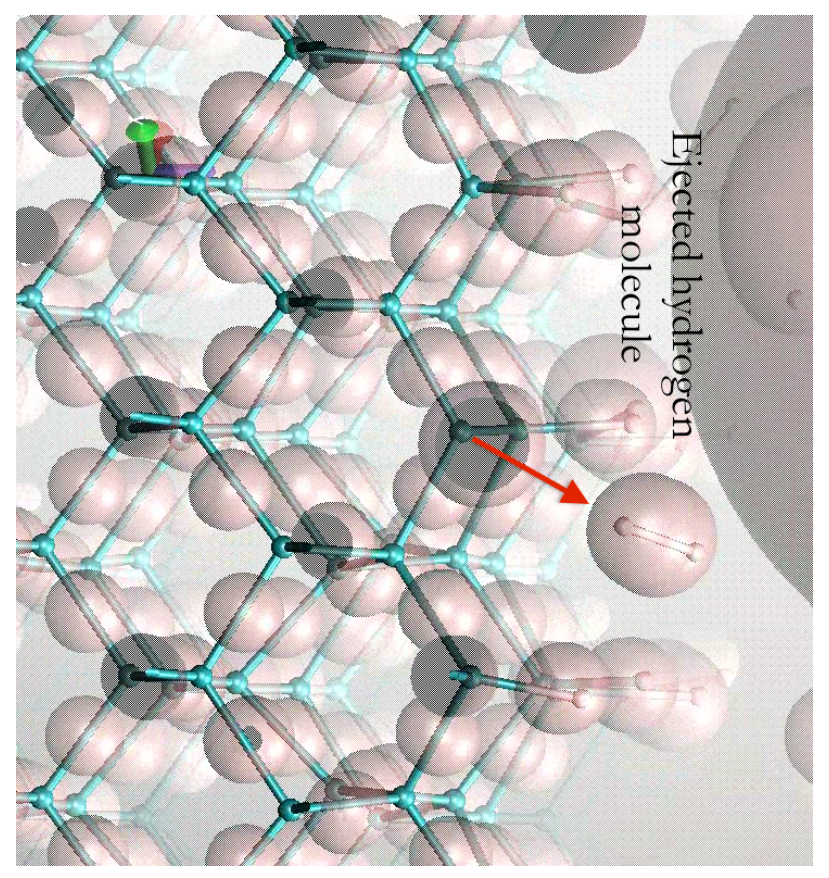

Figure 9: Femtosecond electronic excitation-induced desorption of hydrogen from a passivated diamond slab; a hydrogen molecule is ejected after 80 femtoseconds from surface dimer site (indicated by the red arrow). Electrons are shown as pink transparent spheres of different sizes (including ionized electrons in large spheres).

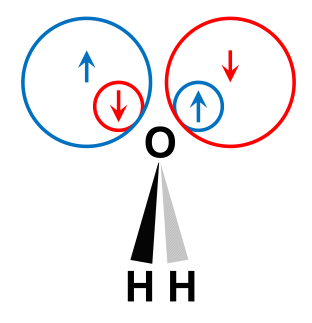

Figure 10: Open shell like representation of lone pairs in eFF-ECP, taking water molecule as an illustration. 


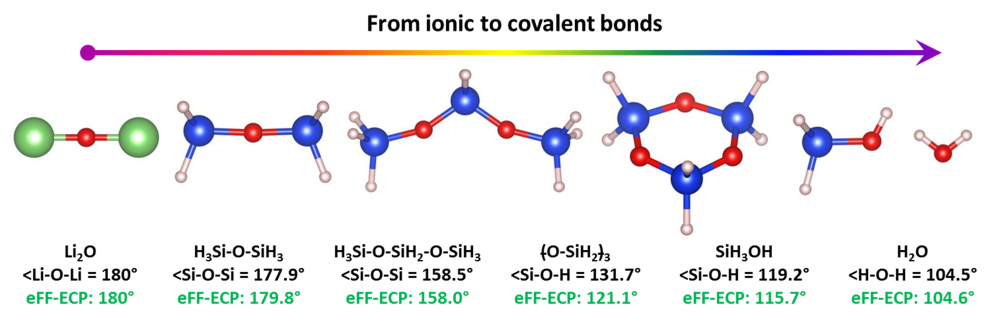

Figure 11: Accurate eFF-ECP geometries from ionic to covalent bonds.

between lone pairs and bonding electrons becomes more dominant, leading to more bent molecular conformations, such as $\mathrm{H}_{2} \mathrm{O}$. Table 4 lists the comparison of optimized geometries from eFF-ECP and experiments, demonstrating the ability of eFF-ECP to describe correctly the trending of oxygen-centered angles and thus the subtle response of lone pairs to varying chemical environments.

Table 4: Performance of eFF-ECP on geometries of various molecules with oxygen atom(s) (see Figure 11).

\begin{tabular}{ccccccc}
\hline $\begin{array}{c}\text { bond length }(\mathrm{pm}) \\
\text { bond type }\end{array}$ & $\begin{array}{c}\mathrm{Li}_{2} \mathrm{O} \\
\mathrm{Li}-\mathrm{O}\end{array}$ & $\begin{array}{c}\mathrm{Si}_{2} \mathrm{H}_{6} \mathrm{O} \\
\mathrm{Si}-\mathrm{O}\end{array}$ & $\begin{array}{c}\mathrm{Si}_{3} \mathrm{H}_{8} \mathrm{O}_{2} \\
\mathrm{Si}-\mathrm{O}\end{array}$ & $\mathrm{Si}_{3} \mathrm{H}_{6} \mathrm{O}_{2}$ & $\mathrm{SiH}_{3} \mathrm{OH}$ & $\mathrm{H}_{2} \mathrm{O}$ \\
\hline $\begin{array}{c}\text { Expt. } \\
\text { eFF-ECP }\end{array}$ & 161.0 & 163.7 & $164.4 / 153.5$ & 165.9 & $\mathrm{Si}-\mathrm{O}$ & $\mathrm{H}-\mathrm{O}$ \\
\hline
\end{tabular}

\subsection{Silicon carbide with combined $s-s$ and $s-p$ eFF-ECP}

We used GARFfield (?) to develop the eFF-ECP force field for $\mathrm{SiC}$ tabulated in Table 5. These were obtained by optimizing the parameters for a $p$-type carbon ECP expression (Eq. 5) along with our previously published $s$-type silicon parameters(?). In this eFF-ECP scheme, both silicon and carbon atoms are represented by a core effective potential and four valence electrons. Since the electrons are represented by spherical Gaussians with a position, size and spin, the parameter optimization for geometries with complex bonding structures is more challenging than that of a conventional 
force field. We used lower and upper parameter range values between 0.5-3 for the ECP-radius, and 0-100.0 for $a, b, c, d, e$.

Table 5: Carbon $s-p$ functional form parameters in the SiC-ECP force field and Silicon $s-s$ parameters from ?.

\begin{tabular}{ccccccc} 
Atom & ECP-radius & $a$ & $b$ & $c$ & $d$ & $e$ \\
\hline $\mathrm{Si}$ & 1.691 & 0.320852 & 2.283269 & 0.814857 & & \\
$\mathrm{C}$ & 0.630348 & 21.344508 & 0.715963 & 0.954384 & 14.261287 & 5.314525
\end{tabular}

We find that the eFF-ECP force field accurately captures the proper lattice constant for $3 \mathrm{C}-\mathrm{SiC}$ Zinc blende, $4.3496 \AA^{\circ}$ at $0 \mathrm{~K}$ and $4.3551 \AA$ at $297 \mathrm{~K}$. The latter was computed from the radial distribution function (RDF) between nuclear pairs and the corresponding coordination number. A 5000 atom 3C-SiC fully periodic cubic unit cell was minimized to a 1e-8 energy difference using conjugate gradient minimization. 100fs of equilibrated NVT molecular dynamics at 297K (controlled a Nose-Hoover thermostat) was run in LAMMPS with the eff/cut pair style (?) to determine the average RDF and coordination number. Experimental results report a value of $4.3596 \AA$ at $297 \mathrm{~K}(?))$.

The results for the Si-C bond, and Si-Si-C, Si-C-C, H-C-H, H-Si-H, CC-Si, C-Si-C, C-Si-Si, Si-C-Si, Si-C-H and C-Si-H angles from the different conformers in the training set are tabulated in Table.6.

In (?) we had applied a prior $\mathrm{SiC}$ eFF-ECP force field to study the hypervelocity impact (HVI) effects of a nanodiamond cluster on a silicon carbide surface. We found that two distinct heat dissipation domains characterize the thermal transport in $\mathrm{SiC}$ during HVI: a stopping domain and the shock wave propagation domain. We bracketed the stopping domain between the time of impact and the reversal of momentum. On the other hand, the shock wave propagation domain is the time the impact shock wave traverses the cell. Energy is dissipated throughout the cell by onto phonon modes that couple with the shock wave. A greater number of electrons are ionized in the stopping phase, and energy is dissipated more effectively by thermal excitation of electrons than by phonon excitation and scattering. We re-ran these simulations with the improved force field and the results do not change the original description of the thermal dissipation in $\mathrm{SiC}$. 
Table 6: Silicon Carbide training set and optimization results using the $s$ - $p$ form ECP for Carbon. All bond lengths in Bohr and angles in degrees.

\begin{tabular}{lcccc} 
Structure & Feature & QM (BY3LYP $)$ & eFF-ECP & \% Error \\
\hline $\mathrm{SiH}_{3} \mathrm{CH}_{3}$ & Si-C bond & 3.5527 & 3.8177 & 7.4594 \\
$\mathrm{SiH}_{2} \mathrm{CH}_{2}$ & Si-C bond & 3.2314 & 4.0231 & 24.5002 \\
$2 \mathrm{SiH}_{2} 2 \mathrm{CH}_{2}$ & Si-C bond & 3.6417 & 3.5643 & 2.1241 \\
$2\left(\mathrm{SiH}_{2} \mathrm{CH}_{2}\right)$ & Si-Si-C angle & 78.500 & 80.1411 & 2.0906 \\
$2\left(\mathrm{SiH}_{2} \mathrm{CH}_{2}\right)$ & Si-C-C angle & 101.5000 & 99.8589 & 1.6168 \\
$2\left(\mathrm{SiH}_{2} \mathrm{CH}_{2}\right)$ & H-C-H angle & 106.5000 & 118.1523 & 10.9411 \\
$2\left(\mathrm{SiH}_{2} \mathrm{CH}_{2}\right)$ & H-Si-H angle & 107.6000 & 113.7215 & 5.6891 \\
$\mathrm{C}_{2} \mathrm{H}_{5} \mathrm{SiH}_{3}$ & C-C-Si angle & 114.1000 & 114.8811 & 0.6846 \\
$2\left(\mathrm{CH}_{3}\right) \mathrm{SiH}_{2}$ & C-Si-C angle & 112.1000 & 116.4574 & 3.8871 \\
$\mathrm{Si}_{2} \mathrm{H}_{5} \mathrm{CH}_{3}$ & C-Si-Si angle & 112.0000 & 112.298 & 0.2662 \\
$2\left(\mathrm{SiH}_{3}\right) \mathrm{CH}_{2}$ & Si-C-Si angle & 115.7000 & 111.8900 & 3.2930 \\
$\mathrm{SiCH}^{\mathrm{CSiH}}$ & Si-C-H angle & 111.3000 & 116.6054 & 4.7667 \\
\hline
\end{tabular}

\section{Conclusions}

We have developed an ECP representation in the framework of the electron force field (eFF) that overcomes important problems associated with the spherical Gaussian basis set used, and enables the study of dynamic phenomena in materials exposed to extreme loading conditions (including mechanical, thermal, radiation, and other). Such loading conditions cause excited state phenomena, which can cause nucleation of damage sites and defects at the atomic scale, irreversible transformations and eventual failure in materials. The ECP enhancements to the all electron eFF enable:

- accurate simulation of higher-Z elements $(\mathrm{Z}>6)$ ), up to p-block (e.g. C, $\mathrm{Si}, \mathrm{O}, \mathrm{SiC}, \mathrm{SiO}_{2}$, among others),

- substantial improvements in the description of multiple bonds (e.g. Carbon) and London dispersions (e.g. graphite),

- accurate description of lone pairs (e.g. Oxygen),

- accurate description of complex binaries (e.g. $\mathrm{SiC}, \mathrm{SiO}_{2}$ ),

- hybrid description of core and valence ionization events, with accurate ionization potentials, in combination with the all electron eFF, 
- modeling the dynamics of large-scale material systems (millions of atoms) subjected to extreme loading condition.

In addition to enabling the simulation of systems with increasingly nonspherical electrons, the eFF-ECP representation leads to a significant reduction in the total number of degrees of freedom and filters out high frequency modes from core electrons. This results in larger integration time steps when solving EOM and enables longer simulation timescales (10's of nanoseconds for systems with millions of atoms in the LAMMPS implementation). The former comes by virtue of removing the high core electron binding energies and the Nyquist-Shannon criterion, more specifically the Nyquist rate, i.e. one must sample at twice the maximum component frequency of the function being sampled. In (?) we demonstrated the scalability up to millions of atoms for the all-electron eFF methodology, hence the reduced computational complexity and simplified model descriptions in the eFF-ECP framework guarantees its scaling capabilities. The eFF-ECP together with a fixedcore representation, which freezes the core electrons for elements $Z>2$, are available along with the all-electron representation in the open source parallel code of eFF in LAMMPS. All the example cases presented here were calculated using this implementation (?).

Remaining challenges in eFF include further extension to $d$-elements (e.g. using angular momentum projection operators), implicit hybridization support, appropriate account for conjugation, and development of new types of ECP with parameters that retain physical meaning. These enhancements will be part of a future publication.

\section{Acknowledgment}

The Authors would like to thank Matthew Fishman at Caltech for his contribution to the diamond surface hydrogen desorption simulations with eFF-ECP for $\mathrm{C} / \mathrm{H}$. This Material is based upon work supported by the Department of Energy National Nuclear Security Administration under Award Number DE-FC52-08NA28613, and the Defense Advanced Research Projects Agency (DARPA) (Grant No.N660011214037, Drs. Tyler McQuade and Anne Fischer).

\section{References}

\section{References}

